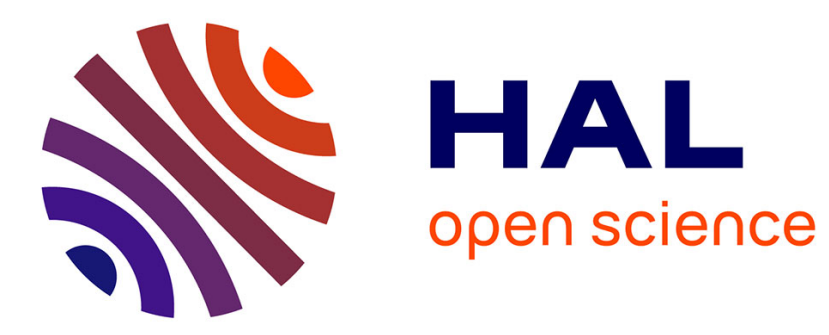

\title{
Modeling the Induction of Psychosocial Stress in Virtual Reality Simulations \\ Celia Kessassi
}

\section{To cite this version:}

Celia Kessassi. Modeling the Induction of Psychosocial Stress in Virtual Reality Simulations. ACIIW 2021: 9th International Conference on Affective Computing and Intelligent Interaction Workshops and Demos, Sep 2021, Nara, Japan. 10.1109/ACIIW52867.2021.9666443 . hal-03561694

\section{HAL Id: hal-03561694 https://hal.science/hal-03561694}

Submitted on 22 Feb 2022

HAL is a multi-disciplinary open access archive for the deposit and dissemination of scientific research documents, whether they are published or not. The documents may come from teaching and research institutions in France or abroad, or from public or private research centers.
L'archive ouverte pluridisciplinaire HAL, est destinée au dépôt et à la diffusion de documents scientifiques de niveau recherche, publiés ou non, émanant des établissements d'enseignement et de recherche français ou étrangers, des laboratoires publics ou privés. 


\title{
Modeling the Induction of Psychosocial Stress in Virtual Reality Simulations
}

\author{
Celia Kessassi \\ DAPI, LS2N, IMT-Atlantique \\ Nantes, France \\ https://orcid.org/0000-0001-6199-7578
}

\begin{abstract}
During the last few years, a wide number of virtual reality applications dealing with psychosocial stress have emerged. However, our current understanding of stress and psychosocial stress in virtual reality hinders our ability to finely control stress induction. In my PhD project I plan to develop a computational model which will describe the respective impact of each factor inducing psychosocial stress, including virtual reality factors, personal factors and other situational factors.
\end{abstract}

Index Terms-Psychosocial stress, Trier Social Stress Test TSST, Threat and challenge theory, computational model

\section{MOTIVATION AND RESEARCH QUESTIONS}

The human-computer interaction field is gaining increasing interest and much effort is invested to optimize interfaces and build more human-centred applications. For instance, O. Carsten and M. H. Martens [1] state that the design and function of the Human Machine Interfaces is crucial for ensuring human safety, this is particularly true in car design. Affective computing, in the other hand, is a special sub-field where the emphasis is put on the detection, understanding and generation of human affect, and where the aim is to develop technologies fulfilling human needs, such as applications providing mental health services in virtual reality [2].

In fact, during the last few years, an increasing number of studies and applications in virtual reality have emerged, some of which, such as social skills training, function by inducing psychosocial stress in virtual environment [3] [4]. Although they were proven to be effective for triggering stress, the mechanism of stress induction is still loosely understood, that is to say, a virtual system that is proven to trigger stress in certain individuals may not induce stress for others. In order to better understand this mechanism, we aim at developing a computational model describing the various parameters in virtual reality affecting psychosocial stress. We believe the work will subsequently contribute to other research domains such as human-machine interactions and stress research in general. Moreover, it will inspire the design of various applications including: social skills training in virtual reality, stress detection, stress prevention, personalized gamification among others.

In order to achieve our goal, we started by defining the problem, that is to say defining what we consider to be stress and psychosocial stress. Among the things we need to take note of are the various parameters impacting stress in virtual interactions, including the external (ex. room size, virtual agent's behaviour..) and internal (ex. personality traits, experience..) parameters. Moreover, virtual reality is a singular environment, different from the real world in term of cognitive impact. Immersion, for instance, plays an important role in the user's behaviour and perceptions [5]. In our research we consider and explore all of these aspects to build our model describing psychosocial stress induction in virtual reality.

\section{BACKGROUND AND RELATED WORK}

\section{A. Definition of stress}

Hans Selye, known as the "father of stress" is considered to be the first to incorporate the term "stress" into the medical lexicon [6] [7] [8] [9] [10]. During his medical school, he observed that patients with a variety of illnesses had many of the same "non-specific" symptoms. To learn more about these symptoms, he conducted a series of experiments where rats were placed under the exposure of various noxious agents, such as cold, surgical injury, production of spinal shock, excessive muscular exercise, or intoxication with sublethal doses of diverse drugs. Consequently, he observed the same symptoms regardless of the nature of the damaging agents.

Stress was described by Selye as "the non-specific response of the body to any demand". In fact, stress is regarded as the universal set of reactions and processes created by an environmental demand. It is designated as non-specific because the body shows the same effect regardless of the source of stress.

\section{B. Psychosocial stress}

Among the many sources of stress, we are interested in the psychosocial stress. Psychosocial stress refers to the stress induced in social relationships or arrangement, and affecting the organism through the mediation of higher nervous processes [11]. According to Blascovich's model of psychosocial stress, also recognized as the BPS model [12], socially stressful situations can fall into two categories, namely threat or challenge. To determine whether the situation constitutes a threat or a challenge, one makes a cognitive appraisal of the situation, if the comparative appraisals results in the perception of situation's demands that can not be overcome with the perceived resources at one's disposal, then threat becomes the overall resulting appraisal. Conversely, if the comparative appraisals results in a perception of resources that can overcome situation's demands, then the challenge is 
the overall resulting appraisal. Demands, as described in the theory, refers to any demand, uncertainty and/or danger.

This threat and challenge theory was influenced by prior work confirming the potential of interpersonal and intrapersonal factors to moderate autonomic responses [12]. In one of Blascovich early experiments, he studied the impact of varied presence of others during a mental arithmetic task. In the experiment, autonomic responses were recorded while participants (middle-age women) performed the task with either a human friend, their pet dog or alone, however the experimenter was present in all three conditions. Results indicated that participants who performed the mental arithmetic task in the presence of their human friend resulted in significantly larger increases in autonomic responses compared to those performing the task with their pet dog. Conversely, the presence of the pet dog induced smaller autonomic responses compared to the presence of the experimenter alone. Blascovich interpreted the results by suggesting that the human friend was more likely to be perceived as evaluative than the canine friend. Thus, interpersonal and intrapersonal factors plays a key role in the level of stress.

Following the development of his theory, he conducted a series of studies that corroborated with the threat and challenge model [13]. For instance, he measured physiological, behavioural and subjective responses of participants interacting with stigmatized partners during a collaborative task. The stigma included facial-birthmarks, race and socioeconomic status. Measures indicated that participants interacting with stigmatized partners exhibited consistent threat responses compared to participants interacting with non-stigmatized partners, who exhibited challenge responses. In addition to the to stereotypes, a similar effect was observed with varied instructions during experiments. The aim of varying instructions was to influence perception of demands by either emphasizing accuracy of task performance and potential evaluation, or doing one's best. As hypothesized threat and challenge responses were consistent with the nature of instructions.

Through those experiments, Blasovich and his colleagues try to provide evidences supporting the BPS model. Moreover, they indicate physiological responses to threat distinct from the physiological responses of challenge, hence, both induce different levels of stress. For instance, results suggests that cardiac output levels increase during challenge compared to threat. In addition to that, self-reported stress levels were observed to be positively related to cognitive appraisal, such that the more threatened one is the more he reports experiencing stress during a task. Although the model has been criticized [14], these findings provide essential information to understand how stress affect the user in general and provide necessary grounds to study psychosocial stress in virtual reality. It is valuable for building the hypotheses and the experimental protocols and it is relevant for establishing measures that indicate stress levels, including physiological measures and self-reported measures.

\section{Stress induction tools}

One of the tools used to trigger stress in laboratory setting is the Trier Social Stress Test (TSST). It's mostly used for its reliability to trigger physiological responses of stress. It consists of an anticipation phase and a speech delivery phase that each lasts 10 minutes. Participants are asked to introduce themselves to a panel of three persons, presented as body language experts, and are told that they would be recorded with a video camera. Participants prepare the speech during the anticipation phase in one room, and they deliver the speech during the speech delivery phase in an other room where the committee and video camera are placed. Following the public speaking phase, the participants are asked to perform a mental arithmetic task in front of the same audience, counting backward from 1022 with steps of 13 .

This protocol is recognized for successfully triggering stress responses [15] [16], including, saliva cortisol increase (70\% of the population respond with an increase of $2.5 \mathrm{nmol} / 1$ above baseline) and heart rate increase.

While the TSST is a very effective tool for inducing psychosocial stress and is valuable as an experimental tool in virtual reality, as it has already been studied [17] [16] [18] [19] [20], our ambition, however, is to identify the characteristics of stressful social situations, in virtual interactions, that can account for different situational contexts, and not only in the context of the TSST protocol.

\section{Stressful situation's features}

In order to explore the different features that makes a situation stressful, L. Lebois et al. [21] carried out an experiment where 12 participants had to evaluate 572 sentences describing life events situation. Participants rated how much those sentences possessed 19 features potentially associated with perceived stressful situations, such as experience or familiarity with the situation. The findings indicates high correlation between perceived stress and some hypothesized features including expectation violation, outcome certainty, negative valence, arousal or coping certainty. These findings are very beneficial since it helps understand the factors correlating with stress, it will be leveraged to study these parameters in virtual reality and help build hypothesis and experimental protocols using varied situations in virtual simulations.

While Lebois and her colleagues studied the features of stressful situations in general, L. B. Fich et al. [16] investigated one particular feature in virtual reality. The study compares the impact of stress between two groups, one group that went through the TSST protocol in a virtual room with openings (three windows in front of the participant) and the other group that participated to the TSST in a virtual room without openings. The hypothesized argument was that in a stressful situation such as public speaking, the physiological responses of stress would be dampened through the presence of windows in the virtual room. This hypothesis stems from the studies suggesting that view through a window influence recovery from surgery. However, apart from saliva cortisol no other biomarker of stress (ex. heart rate, T-wave amplitude) was 
significantly influenced by the presence nor the absence of windows. These suggestions provide an insight on how virtual parameters might induce particular stress patterns.

\section{HYPOTHESIS AND THE SCOPE OF THE TECHNICAL PROBLEM}

At the present moment, a wide range of hypothesis are being considered for the project. Main ones are described in the following.

\section{A. Situational factors and stress responses}

One of the directions we consider is leveraging Blascovich's BPS theory to define the factors that explains stress in virtual reality. As it was described above in section II-B, the effect of particular parameters was investigated in vivo, including varied stereotypes and instructions. Going further, these parameters can also be studied in virtual reality simulations.

Some studies on social exclusion [22] [23] explored the impact of social exclusion on psychological state and stress. One protocol that is frequently used is a virtual ball-tossing game called Cyberball, it is a standardized tool for social exclusion simulation. The virtual game counts 3 players, one that represents and can be controlled by the participant and the others represent two other players. The players has to toss the ball to one of the other players, and at a particular moment, the two other player stop tossing the ball to the participant. Results shows significant effect on mood, and even neurological evidences of social pain, however, Weik and his colleagues [23] reported no effect on saliva cortisol, which is one of stress biomarkers. These findings raises questions on the nature and type of demands that triggers stress. One may speculate that this social exclusion experiment create situational demands that exceeds appraised resources, consequently, according to the BPS model, stress and in particular threat should be experienced. Nevertheless, this account contradicts with experimental results. Thus, in order to predict how a virtual social interaction affects stress, a full and comprehensive understanding of the appraisal of demand and resources is required. For this reason, we envisage the possibility to explore more about cues impacting the perception of demands and perception of the resources at one's disposal.

Despite all, the endeavour of studying the nature of demands and resources appraisal and how they combine seem challenging. According to Blaschovich the "appraisal" process is influenced by multivariate and multi-process systems including conscious and unconscious processes, and the complex influence of conscious and unconscious on the relationship between demand and resources can not be obtained with self-reported data [24].

\section{B. Perspective as a factor}

According to previous research related to perspective (ex. first-person perspective) in virtual reality, the perspective from which one observes a situation determines the way he experiences it [25] [26]. For instance, Bergström and his colleagues suggest that observing a situation from the first-person rather than the third-person perspective leads to high subjective body ownership illusion, which consequently leads to higher stress responses in uncomfortable virtual body posture situation [27]. In contrast, another study suggests that higher degrees of anxiety appear to be linked to increased third-person perspective in social phobia [28], moreover, Clark and Wells propose that individuals with social phobia view themselves in a negative impression of how they appear to others, as the observer perspective [29]. These suggestions point out the probable relevance of perspective to influence social stress. However, whether psychosocial stress level is more important in first-person or third-person perspective is not certain yet, more extensive research should be conducted to answer these questions.

Another hypothesis related to the suggestions above involves the potential of body ownership illusion to influence stress. For instance, we consider maximizing the body ownership illusion using other techniques, such as using a virtual 3D scanned version of the person and analyze the impact on social stress.

\section{Mutiplicy of stressors in the TSST}

Common findings demonstrated the reliably of the TSST to induce physiological responses of stress, however, the protocol is not limited to a public speaking task, it includes an apprehension phase, that can in itself be a stressor because it creates demands for anticipation and preparation, more importantly, it creates uncertainty that may lead to experiencing threat. In addition, the mental arithmetic phase is a mental task that can introduce heart rate increase which is not related to psychosocial stress, because mental demanding tasks are known to alter cardiovascular responses [30]. In the other hand, the unexpected nature of the mental task can be the source of the stress response. Studying the respective effect of these phases might provide a better insight about which situation will be perceived as socially stressful or non-stressful in the context of the TSST protocol.

\section{OBJECTIVES AND METHODOLOGY}

To summarize, our goal is to understand the various factors in a given virtual social interaction situation impacting stress responses and determine, according to these factors, how stress bio-markers are impacted. Based on the literature review, there is 3 main components that are essential for the model describing psychosocial stress in virtual reality (see fig. 1). First, one main component is the virtual reality parameters. As mentioned above, many studies report significant effect on psychosocial stress while virtual system parameters were varied. These parameters may include the virtual architectural design, perspective, the audience size or audience behaviour [31] [4] [16]. The second component of the model is the situational parameters. We believe that the situational settings in which someone experiences a stressful situation plays a key role, for instance, maybe if someone experiences a stressful situation in laboratory settings rather than at home, maybe stress wouldn't be experienced the same way. Finally, there 
is the component encompassing personal factors. As it has been supported from a wide number of theories and research studies, this component seems to be the key factor that dictates, depending on the individual, if a particular situation is perceived as stressful or non-stressful . It may include personal factors such as past experience, personal state or personal traits.

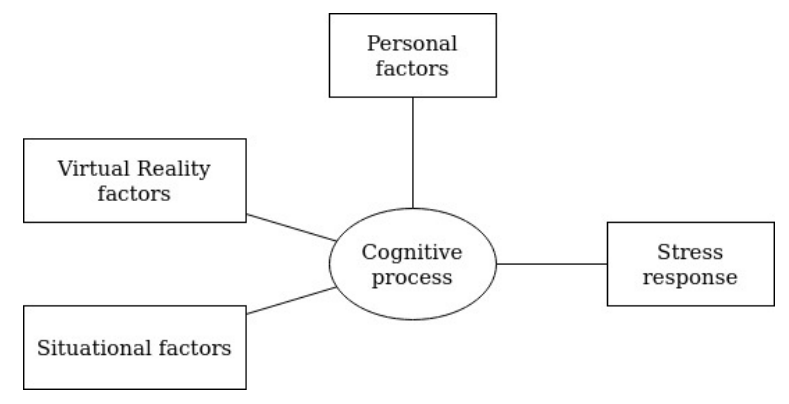

Fig. 1. An overview of the components under study for stress induction

The model we aim to develop is also a model that is able to predict stress responses according to various parameters, doing so requires understanding the respective impact of each parameter. In addition, we have seen in a number of studies where varying a parameter such as the presence of windows in virtual environment has an impact on some but not all biomarkers of stress. It suggests that different stressors will induce different stress patterns.

Accordingly, we will continue reviewing the literature related to factors of stressful situation, with the purpose of collecting information and determining which parameter is relevant to be incorporated in the model and which pattern of stress is induced.

If one parameter is not sufficiently explored, we shall endeavour to study it further. This should be completed by means of experimental studies where we will vary virtual and situational parameters and analyze the stress response.

Main stress responses may be investigated through perceptual, behavioural, and physiological responses. The evaluation of perceptual responses to a stressor involves subjective estimations and perceptions. Indeed, to measure self-reported stress level, numerous questionnaires have been used in research, including the Cohen's Perceived Stress Scale (PSS) that measures the degree to which situations are considered stressful, doing so by addressing events experienced in the preceding month [32]. Another one is the Strain Questionnaire (SQ) a multi-model perception of stress that measures behavioral, cognitive, and physical stress perception of stress [33].

Regarding the physiological responses of stress, saliva cortisol along with heart rate are two of stress biomarkers frequently investigated. The more stressed someone is, the more cortisol and heart rate increase. Prior studies demonstrated that stress has also electroencephalogram correlates, thus, it can indicate stress level [34]. Moreover Elevated skin conductance is found to be correlated with stress elevation [35]. The above described biomarkers will be important biomarkers to assess stress responses during experiments.

Finally, the goal is to build a reliable model. Its reliability will have to be validated using experimental data, doing so by comparing the model with the experimental data.

\section{A DESCRIPTION OF THE WORK DONE SO FAR AND A TENTATIVE PLAN FOR FUTURE WORK}

As the $\mathrm{PhD}$ project started only recently, much attention is currently being paid to literature and establishing the list of parameters relevant to the model. Once the literature has been sufficiently explored the subject should be narrowed down and clear hypothesis should be formulated. From there, we will develop an experimental framework for virtual interactions where the parameters under study will be varied to analyze their impacts. These parameters might include the virtual room design, or the instructions given to the participants, it may include a variety of parameters, however, at the present moment there is no definite parameter, as our hypotheses may evolve. After the development of the virtual simulation framework, we plan to carry out experiments and collect data that will serve to study our hypothesis and to develop the computational model.

\section{EXPECTED CONTRIBUTIONS}

We hope that our work will contribute to provide a thorough understanding of the mechanisms underlying psychosocial stress induction in virtual reality simulations. Such understanding will have impacts in the field of virtual reality in general, and in human-agent interactions in particular, as this will further our knowledge about the factors shaping user's experience during virtual social interactions. Furthermore, the model might serve as support to build, using the appropriate characteristic, applications that need full control of stress induction.

The aimed computation model should be able to detect, based on stress responses of the user, if the corresponding virtual-social interaction is appraised as stressful or nonstressful. Moreover, it will be able to predict, based on the user's personal factors, if a particular virtual-social interaction will be perceived as stressful. The methods, tools and results produced in the project will enable the creation of experiments to test hypotheses and make predictions which will in turn contribute to progress in social and cognitive psychology.

Finally, the experiments we intend to conduct will lead to the creation of a multimodal corpora that will be made available to the scientific community, after a strict audit of the data, ensuring that the anonymisation procedure was carried out properly. These corpora will be invaluable tools for further research on virtual social interactions, social stress induction mechanisms, and computational models applied to social behaviour and physiological signals. 


\section{REFERENCES}

[1] O. Carsten and M. H. Martens, "How can humans understand their automated cars? hmi principles, problems and solutions," Cognition, Technology \& Work, vol. 21, no. 1, pp. 3-20, 2019.

[2] M. J. Park, D. J. Kim, U. Lee, E. J. Na, and H. J. Jeon, "A literature overview of virtual reality (vr) in treatment of psychiatric disorders: recent advances and limitations," Frontiers in psychiatry, vol. 10, p. 505, 2019.

[3] T. D. Parsons and A. A. Rizzo, "Affective outcomes of virtual reality exposure therapy for anxiety and specific phobias: A meta-analysis," Journal of behavior therapy and experimental psychiatry, vol. 39, no. 3 , pp. 250-261, 2008.

[4] D.-P. Pertaub, M. Slater, and C. Barker, "An experiment on public speaking anxiety in response to three different types of virtual audience," Presence: Teleoperators \& Virtual Environments, vol. 11, no. 1, pp. 6878, 2002.

[5] A. Alshaer, H. Regenbrecht, and D. O'Hare, "Immersion factors af fecting perception and behaviour in a virtual reality power wheelchair simulator," Applied ergonomics, vol. 58, pp. 1-12, 2017.

[6] S. Y. Tan and A. Yip, "Hans selye (1907-1982): Founder of the stress theory," Singapore medical journal, vol. 59, no. 4, p. 170, 2018.

[7] G. Fink, "Stress: Concepts, definition and history," in Reference Module in Neuroscience and Biobehavioral Psychology, Elsevier, 2017.

[8] R. S. Lazarus and S. Folkman, Stress, appraisal, and coping. Springer publishing company, 1984.

[9] H. Selye, "Confusion and controversy in the stress field," Journal of human stress, vol. 1, no. 2, pp. 37-44, 1975.

[10] H. Selye, "Forty years of stress research: principal remaining problems and misconceptions.," Canadian Medical Association Journal, vol. 115, no. 1, p. 53, 1976.

[11] L. Levi, Stress and Distress in Response to Psychosocial Stimuli: Laboratory and Real-Life Studies on Sympatho-Adrenomedullary and Related Reactions. Elsevier Science, 2016.

[12] J. Blascovich and J. Tomaka, "The biopsychosocial model of arousal regulation," Advances in experimental social psychology, vol. 28, pp. 1$51,1996$.

[13] J. Tomaka, J. Blascovich, J. Kibler, and J. M. Ernst, "Cognitive and physiological antecedents of threat and challenge appraisal.," Journal of Personality and Social Psychology, vol. 73, no. 1, pp. 63-72, 1997.

[14] R. A. Wright and L. D. Kirby, "Cardiovascular correlates of challenge and threat appraisals: A critical examination of the biopsychosocial analysis," Personality and Social Psychology Review, vol. 7, no. 3, pp. 216-233, 2003.

[15] C. Kirschbaum, K.-M. Pirke, and D. H. Hellhammer, "The 'trier social stress test'-a tool for investigating psychobiological stress responses in a laboratory setting," Neuropsychobiology, vol. 28, no. 1-2, pp. 76-81, 1993.

[16] L. B. Fich, P. Jönsson, P. H. Kirkegaard, M. Wallergård, A. H. Garde, and $\AA$. Hansen, "Can architectural design alter the physiological reaction to psychosocial stress? a virtual tsst experiment," Physiology \& behavior, vol. 135, pp. 91-97, 2014.

[17] E. Montero-López, A. Santos-Ruiz, M. C. García-Ríos, R. RodríguezBlázquez, M. Pérez-García, and M. I. Peralta-Ramírez, "A virtual reality approach to the Trier Social Stress Test: Contrasting two distinct protocols," Behavior Research Methods, vol. 48, pp. 223-232, Mar. 2016.

[18] P. Zimmer, B. Buttlar, G. Halbeisen, E. Walther, and G. Domes, "Virtually stressed? A refined virtual reality adaptation of the Trier Social Stress Test (TSST) induces robust endocrine responses," Psychoneuroendocrinology, vol. 101, pp. 186-192, Mar. 2019.

[19] C. C. Vatheuer, A. Vehlen, B. von Dawans, and G. Domes, "Gaze behavior is associated with the cortisol response to acute psychosocial stress in the virtual tsst," Journal of Neural Transmission, pp. 1-10, 2021.

[20] Q. Liu and W. Zhang, "Sex differences in stress reactivity to the trier social stress test in virtual reality," Psychology Research and Behavior Management, vol. 13, p. 859, 2020.

[21] L. A. Lebois, C. Hertzog, G. M. Slavich, L. F. Barrett, and L. W. Barsalou, "Establishing the situated features associated with perceived stress," Acta Psychologica, vol. 169, pp. 119-132, 2016.

[22] C. Zöller, P. Maroof, U. Weik, and R. Deinzer, "No effect of social exclusion on salivary cortisol secretion in women in a randomized controlled study," Psychoneuroendocrinology, vol. 35, no. 9, pp. 1294 1298, 2010.

[23] U. Weik, P. Maroof, C. Zöller, and R. Deinzer, "Pre-experience of social exclusion suppresses cortisol response to psychosocial stress in women but not in men," Hormones and Behavior, vol. 58, no. 5, pp. 891-897, 2010.

[24] J. Blascovich, W. B. Mendes, J. Tomaka, K. Salomon, and M. Seery, "The Robust Nature of the Biopsychosocial Model Challenge and Threat: A Reply to Wright and Kirby," Personality and Social Psychology Review, vol. 7, pp. 234-243, Aug. 2003.

[25] P. Salamin, D. Thalmann, and F. Vexo, "The benefits of third-person perspective in virtual and augmented reality?," in Proceedings of the ACM symposium on Virtual reality software and technology, pp. 27-30, 2006.

[26] D. Monteiro, H.-N. Liang, A. Abel, N. Bahaei, and R. d. C. Monteiro, "Evaluating engagement of virtual reality games based on first and third person perspective using eeg and subjective metrics," in 2018 IEEE International Conference on Artificial Intelligence and Virtual Reality (AIVR), pp. 53-60, 2018.

[27] I. Bergström, K. Kilteni, and M. Slater, "First-person perspective virtual body posture influences stress: a virtual reality body ownership study," PloS one, vol. 11, no. 2, p. e0148060, 2016.

[28] S. M. A. Wallace-Hadrill and S. K. Kamboj, "The Impact of Perspective Change As a Cognitive Reappraisal Strategy on Affect: A Systematic Review," Frontiers in Psychology, vol. 7, 2016.

[29] J. M. Spurr and L. Stopa, "The observer perspective: effects on social anxiety and performance," Behaviour Research and Therapy, vol. 41, no. 9, pp. 1009-1028, 2003.

[30] R. Sloan, J. Korten, and M. Myers, "Components of heart rate reactivity during mental arithmetic with and without speaking," Physiology \& Behavior, vol. 50, pp. 1039-1045, Nov. 1991.

[31] F. Mostajeran, M. B. Balci, F. Steinicke, S. Kühn, and J. Gallinat, "The effects of virtual audience size on social anxiety during public speaking," in 2020 IEEE Conference on Virtual Reality and 3D User Interfaces (VR), pp. 303-312, IEEE, 2020.

[32] S. Cohen, T. Kamarck, and R. Mermelstein, "A global measure of perceived stress," Journal of health and social behavior, pp. 385-396, 1983.

[33] R. C. Lefebvre and S. L. Sandford, "A multi-modal questionnaire for stress," Journal of Human Stress, vol. 11, no. 2, pp. 69-75, 1985.

[34] N. M. Ehrhardt, J. Fietz, J. Kopf-Beck, N. Kappelmann, and A.-K. Brem, "Separating eeg correlates of stress: Cognitive effort, time pressure, and social-evaluative threat," European Journal of Neuroscience, 2021.

[35] C. Jeunet., F. Lotte., and C. Mühl., "Design and validation of a mental and social stress induction protocol - towards load-invariant physiologybased stress detection," in Proceedings of the International Conference on Physiological Computing Systems - PhyCS, pp. 98-106, INSTICC, SciTePress, 2014 